\title{
A POLYNOMIAL TIME SPECTRAL DECOMPOSITION TEST FOR CERTAIN CLASSES OF INVERSE M-MATRICES*
}

\author{
JEFFREY L. STUART ${ }^{\dagger}$
}

Dedicated to Hans Schneider on the occasion of his seventieth birthday

Abstract. The primary result in this paper is a set of $O\left(n^{3}\right)$ time algorithms to determine whether a specified real, symmetric matrix is a member of any of several closely related classes: the MMA-matrices; the inverse MMA-matrices; the strictly positive, positive definite matrices whose eigenvectors form a Soules basis; and the strictly ultrametric matrices. When the class membership question is answered in the affirmative for an MMA-matrix or an inverse MMA-matrix, the algorithms also yield the complete spectral decomposition of the matrix in question. Additional results in this paper include an algorithmic test for when a matrix is a Soules matrix, and a construction for Soules bases.

AMS subject classifications. 15A48, 15A 18

Key words. MMA-matrix, inverse M-matrix, Soules matrix, strictly ultrametric matrix

1. Background. In [3], Friedland, Hershkowitz and Schneider introduced a new matrix construction process, which they called inflation, and a new subclass of the M-matrices, which they called the MMA-matrices. Using the process of inflation, they characterized the MMA-matrices in terms of an inflation sequence. In [2], Fiedler developed an alternative characterization of the MMA-matrices in terms of the sums of their spectral projectors. In [10] and [14], Schneider and Stuart developed a combinatorial relationship between the spectral projectors of an MMA-matrix, and produced a graph-theoretic interpretation of that relationship. In several further papers [11],[12], [13], Stuart elucidated the internal structure of spectral projectors produced via inflation, and provided a polynomial time algorithm for detecting when a matrix is an MMA-matrix.

In [9], Soules presented a class of real, symmetric matrices with nonnegative spectra and a strictly positive eigenvector. In [1], Elsner, Nabben and Neumann produced a construction for all matrices of the type that Soules had investigated, and presented a graph-theoretic structure for that construction.

Martinez, Michon and San Martín introduced a class of matrices that they called the strictly ultrametric matrices in [5]. These matrices were later investigated in several other papers [6],[7],[8]. In [1], Elsner, Nabben and Neumann proved that there was a simple bijection between the class of strictly ultrametric matrices and the class of inverse MMA-matrices.

2. The Matrix Classes And Their Interrelationships. Throughout this paper, all matrices will be $n \times n$, real matrices unless otherwise specified. A matrix or a vector is called strictly positive if all of its entries are positive. Although some

*Received by the editors on 1 December 1997. Final manuscript accepted on 13 August 1998. Handling editor: Daniel Hershkowitz.

${ }^{\dagger}$ Department of Mathematics, University of Southern Mississippi, Hattiesburg, Mississippi 394065045, USA (jeff.stuart@usm.edu). 
authors allow M-matrices to include the singular case, M-matrices will always be nonsingular unless otherwise specified.

The matrix $A$ is called an MMA-matrix if each positive integer power of $A$ is an irreducible M-matrix. In [3], it was proven that if $A$ is a nonsingular MMA-matrix, then $A$ is diagonalizable with real spectrum $\left\{\lambda_{1}, \lambda_{2}, \ldots, \lambda_{n}\right\}$ satisfying $0<\lambda_{1}<$ $\lambda_{2} \leq \cdots \leq \lambda_{n}$, and that there are strictly positive row and column eigenvectors corresponding to the simple eigenvalue $\lambda_{1}$. It was also proven that if $A$ is an MMAmatrix, then there exists a diagonal matrix $D$ with strictly positive diagonal such that $D^{-1} A D$ is a symmetric MMA-matrix. Indeed, in [4], one choice of $D$ is explicitly determined as $D=\operatorname{diag}\left(x_{1}^{1 / 2} y_{1}^{-1 / 2}, x_{2}^{1 / 2} y_{2}^{-1 / 2}, \ldots, x_{n}^{1 / 2} y_{n}^{-1 / 2}\right)$, where $x$ and $y$ are, respectively, the normalized, strictly positive, right and left eigenvectors of $A$.

The matrix $B$ is called an inverse MMA-matrix if $B^{-1}$ is an MMA-matrix. (In [2], the inverse MMA-matrices were called $\mathrm{M}^{-1} \mathrm{MA}$-matrices.) Note that since the inverse of an irreducible M-matrix is strictly positive, it follows that every positive integer power of an inverse MMA-matrix must be strictly positive.

The real, orthogonal matrix $R$ is called a Soules matrix (so named in [1]) if the first column of $R$ is strictly positive and if $R \Sigma R^{T}$ is a nonnegative matrix for every matrix $\Sigma$, where $\Sigma=\operatorname{diag}\left(\sigma_{1}, \sigma_{2}, \ldots, \sigma_{n}\right)$ with $\sigma_{1} \geq \sigma_{2} \geq \cdots \geq \sigma_{n} \geq 0$. When $R$ is a Soules matrix, the set of columns of $R$ is called a Soules basis for $\mathbb{R}^{n}$. In remarks following Observation 2.1 in [1], it was argued that if $A=R \Sigma R^{T}$, where $R$ is a Soules matrix and $\Sigma$ has the indicated properties, then $A$ is irreducible if and only if $\sigma_{1}>\sigma_{2}$, in which case, $A$ is strictly positive. In light of Observation 2.1 in [1], Fiedler's Theorem 1 in [2] can be interpreted as stating that an orthonormal set of eigenvectors of an inverse MMA-matrix form a Soules basis. Elsner, Nabben and Neumann observe that the converse holds [1, end of Section 2]. Thus:

THEOREM 2.1. Let $A$ be a nonsingular, symmetric, $n \times n$ real matrix. The following are equivalent:

1. A is an MMA-matrix;

2. $A^{-1}$ is an inverse MMA-matrix;

3. $A^{-1}=R \Sigma R^{T}$, where $R$ is a Soules matrix and $\Sigma=\operatorname{diag}\left(\sigma_{1}, \sigma_{2}, \ldots, \sigma_{n}\right)$ with $\sigma_{1}>\sigma_{2} \geq \cdots \geq \sigma_{n}>0$.

The symmetric, nonnegative matrix $A$ is called a strictly ultrametric matrix if $A=\left[a_{i j}\right]$ satisfies a pair of "metric" properties:

(i) $a_{i j} \geq \min \left\{a_{i k}, a_{k j}\right\}$ for all $i, j, k \in\langle n\rangle$;

(ii) $a_{i i}>\max \left\{a_{i k} \mid k \in\langle n\rangle \backslash\{i\}\right\}$ for all $i \in\langle n\rangle$;

where $\langle n\rangle=\{1,2, \ldots, n\}$.

Martínez, Michon and San Martín proved in [5] that the inverse of a strictly ultrametric matrix is a symmetric, diagonally dominant M-matrix. Consequently, if $e$ is the $n \times 1$ vector of ones, and if $A$ is a strictly ultrametric matrix, then $p=A^{-1} e$ must be strictly positive.

The final results of this section relate the class of symmetric, inverse MMAmatrices and the class of irreducible, strictly ultrametric matrices. These are theorems 3.1 and 3.2 of [1], and together they give a bijection between the two classes.

THEOREM 2.2. Let $A$ be a symmetric, inverse MMA-matrix. Let $x$ be the normalized, strictly positive eigenvector of $A$. Let $D=\operatorname{diag}\left(x_{1}, x_{2}, \ldots, x_{n}\right)$. Then DAD 
is a strictly ultrametric matrix.

THEOREM 2.3. Let $A$ be an irreducible, strictly ultrametric matrix. Let $p=A^{-1} e$, where $e$ is the vector of ones. Let $F=\operatorname{diag}\left(p_{1}^{1 / 2}, p_{2}^{1 / 2}, \ldots, p_{n}^{1 / 2}\right)$. Then $F A F$ is a symmetric, inverse MMA-matrix.

3. Inflation and MMA-Matrices. Throughout this section and in all following sections we will employ the notation and definitions related to inflation that were introduced for matrices in [3] and extended to vectors in [12]. In particular, $\times \times$ will denote the inflation product (with respect to some partition $\Pi$ ), $\left\{U_{i}\right\}_{i=1}^{k}$ will denote a sequence of strictly positive inflators, and $G(U)$ will denote the idempotent matrix associated with the inflator $U$. Note that every inflator is a rank one matrix, but an inflator $U$ is called a rank one inflator precisely when $G(U)$ has rank one. Finally, when it exists, $A / / U$ will denote the unique matrix $B$ such that $A=B \times \times U$.

One of the principal results of [3] concerned expressing an MMA-matrix in terms of a smaller order MMA-matrix via the process of inflation using some strictly positive inflator. That result was refined in [12], where it was shown that the inflator could be chosen to be a rank one inflator. Summarizing:

THEOREM 3.1. Let $A$ be an $n \times n$ real matrix for some $n \geq 2$. The matrix $A$ is an MMA-matrix if and only if there exists a strictly positive, rank one inflator $U$ such that $A=B \times \times U+\rho G(U)$, where $B$ is an $(n-1) \times(n-1) M M A$-matrix and $\rho$ is the spectral radius of $A$. Furthermore, $\operatorname{spec}(A)=\operatorname{spec}(B) \cup\{\rho\}$ as a multiset. Finally, the matrix $A$ is symmetric if and only if both $B$ and $U$ are symmetric.

Friedland, Hershkowitz and Schneider also derived a spectral decomposition result for MMA-matrices in terms of inflation; see [3]. That decomposition was refined in terms of rank one inflators in [12], and the following result is a special case of the "Weak Slide Around Theorem" (Theorem 7.2) of [10]:

THEOREM 3.2. Let $A$ be an $n \times n$ MMA-matrix with spectrum $\left\{\lambda_{1}, \lambda_{2}, \ldots, \lambda_{n}\right\}$ satisfying $0<\lambda_{1}<\lambda_{2} \leq \cdots \leq \lambda_{n}$. Then there is an inflation sequence $\left\{U_{k}\right\}_{k=1}^{n}$ of strictly positive, rank one inflators such that

$$
A=\sum_{k=1}^{n} \lambda_{k} E_{k}
$$

where the $E_{k}$ are pairwise orthogonal, rank one, idempotent matrices determined by

$$
E_{k}=G\left(U_{k}\right) \times \times U_{k+1} \times \times \cdots \times U_{n}
$$

for $1 \leq k<n$, and

$$
E_{n}=G\left(U_{n}\right)
$$

Finally, we close this section with some basic properties of inflation from [3] that will prove useful.

LEMMA 3.3. Let $U$ be a strictly positive inflator. Let $B$ be a square matrix such that $B \times \times U$ is defined. Let $\lambda$ be a nonzero scalar. Then,

i. $(B \times \times U) G(U)=G(U)(B \times \times U)=0$;

ii. $G(U)$ is an idempotent, singular $M$-matrix;

iii. If $B$ is invertible, and if $A=B \times \times U+\lambda G(U)$, then $A^{-1}=B^{-1} \times \times U+\lambda^{-1} G(U)$. 
4. Sparse Eigenvectors for MMA-matrices. In theorems 12.1 and 13.1 of [13], it was proven that if $A$ is an MMA-matrix, then $\rho(A)$, the spectral radius of $A$, was actually an eigenvalue not just of $A$, but also of some $2 \times 2$ principal submatrix of $A$, and that the row and column eigenvectors of some such principal submatrix of $A$ extended to eigenvectors of $A$ by being embedded in zero vectors of the appropriate size. Here we prove a stronger result.

THEOREM 4.1. Let $A$ be an $n \times n$ MMA-matrix for some $n \geq 2$. Then

$$
\begin{aligned}
\rho(A) & =\max _{1 \leq i<j \leq n} \rho(A[\{i, j\}]) \\
& =\max _{1 \leq i<j \leq n} \frac{1}{2}\left[a_{i i}+a_{j j}+\left[\left(a_{i i}-a_{j j}\right)^{2}+4 a_{i j} a_{j i}\right]^{\frac{1}{2}}\right]
\end{aligned}
$$

and furthermore, if $\{i, j\}$ is any pair of indices for which the maximum occurs, then there exist row and column eigenvectors of $A$ for $\rho(A)$ for which the only nonzero entries are exactly the $i$ and $j$ entries, and those vectors are unique up to scalar multiples. [13].

Proof. The equivalence of the two expressions for $\rho(A)$ is from Theorem 13.1 of

As noted previously, if $A$ is an MMA-matrix, then $A$ is diagonally symmetrizable. Since diagonal symmetrizability carries over to principal submatrices and preserves the spectra of principal submatrices, it suffices to consider the case where $A$ is symmetric.

By Theorem 6.18 of [3], $A=B \times \times U+\rho G(U)$, where $B$ is a symmetric MMAmatrix, $\rho$ is the spectral radius of $A, \rho(B)<\rho$, and $U$ is a symmetric, strictly positive inflator. Since $B$ is a real, symmetric matrix, the Cauchy interlacing inequalities apply to its spectrum. In particular, for all distinct indices $\alpha$ and $\beta$,

$$
\rho(B) \geq \lambda_{\max }\left(\left[\begin{array}{ll}
b_{\alpha \alpha} & b_{\alpha \beta} \\
b_{\beta \alpha} & b_{\beta \beta}
\end{array}\right]\right) .
$$

Since $\rho>\rho(B)$, it follows that $\rho^{2}-\left(b_{\alpha \alpha}+b_{\beta \beta}\right) \rho+\left(b_{\alpha \alpha} b_{\beta \beta}-b_{\alpha \beta}^{2}\right)>0$. Equivalently, $\left(\rho-b_{\alpha \alpha}\right)\left(\rho-b_{\beta \beta}\right)>b_{\alpha \beta}^{2}$. And hence, for any nonzero constants $c$ and $d$,

$$
\left[\left(\rho-b_{\alpha \alpha}\right) c^{2}-\left(\rho-b_{\beta \beta}\right) d^{2}\right]^{2}+4 b_{\alpha \beta}^{2} c^{2} d^{2}<\left[\left(\rho-b_{\alpha \alpha}\right) c^{2}+\left(\rho-b_{\beta \beta}\right) d^{2}\right]^{2} .
$$

Also by the Cauchy interlacing inequalities, $\rho>b_{\alpha \alpha}$ and $\rho>b_{\beta \beta}$.

Now suppose that $\{i, j\}$ is any pair of indices for which the maximum occurs. That is, $\rho=\rho(A[\{i, j\}])$. Since $U$ is a symmetric inflator, $U=u u^{t}$ for some strictly positive vector $u$, and since $A=B \times \times U+\rho G(U)$, there are indices $\alpha$ and $\beta$ such that $a_{i i}=b_{\alpha \alpha} U_{i i}+\rho\left(1-U_{i i}\right)=\rho-\left(\rho-b_{\alpha \alpha}\right) u_{i}^{2}, a_{j j}=\rho-\left(\rho-b_{\beta \beta}\right) u_{j}^{2}$, and $a_{i j}=$ $a_{j i}=\left(b_{\alpha \beta}-\delta \rho\right) u_{i} u_{j}$, where $\delta=1$ when $\alpha=\beta$, and $\delta=0$ otherwise.

Suppose that $\alpha \neq \beta$. Then

$$
\begin{aligned}
\rho & =\frac{1}{2}\left[a_{i i}+a_{j j}+\left[\left(a_{i i}-a_{j j}\right)^{2}+4 a_{i j}^{2}\right]^{\frac{1}{2}}\right] \\
& =\rho-\frac{1}{2}\left[\left(\rho-b_{\alpha \alpha}\right) u_{i}^{2}+\left(\rho-b_{\beta \beta}\right) u_{j}^{2}\right]+\frac{1}{2}\left[\left[\left(\rho-b_{\alpha \alpha}\right) u_{i}^{2}-\left(\rho-b_{\beta \beta}\right) u_{j}^{2}\right]^{2}+4 b_{\alpha \beta}^{2} u_{i}^{2} u_{j}^{2}\right]^{\frac{1}{2}}
\end{aligned}
$$


Setting $c=u_{i}$ and $d=u_{j}$, and using the positivity of $\left(\rho-b_{\alpha \alpha}\right) c^{2}+\left(\rho-b_{\beta \beta}\right) d^{2}$, the previously derived inequality yields:

$$
\rho<\rho-\frac{1}{2}\left[\left(\rho-b_{\alpha \alpha}\right) u_{i}^{2}+\left(\rho-b_{\beta \beta}\right) u_{j}^{2}\right]+\frac{1}{2}\left[\left(\rho-b_{\alpha \alpha}\right) u_{i}^{2}+\left(\rho-b_{\beta \beta}\right) u_{j}^{2}\right]=\rho,
$$

a contradiction. Thus $\alpha=\beta$. That is, $u_{i}$ and $u_{j}$ are both from the $\alpha^{\text {th }}$ partition block of $u$. Let the vector $w$ be obtained from the $n \times 1$ zero vector by replacing the $i^{t h}$ entry with $u_{j}$ and the $j^{\text {th }}$ entry with $-u_{i}$. Clearly $w^{t} u=0$, and hence, by theorems 5.1 and 6.6 of [11], it follows that $w$ is a column eigenvector of $A$ for $\rho$ with the desired properties. Also, from Theorem 5.1 of [11], $w$ is, up to scalar multiplication, the unique eigenvector with precisely the $i^{\text {th }}$ and $j^{\text {th }}$ entries nonzero.

5. Testing for MMA-matrices. In this section, we do not require the matrix $A$ to be symmetric.

THEOREM 5.1. The following algorithm determines whether the given $n \times n$, real matrix $A$ is an MMA-matrix. The algorithm requires at most $O\left(n^{3}\right)$ multiplications and divisions, and when $A$ is an MMA-matrix, the algorithm produces the spectral decomposition of $A$.

Algorithm 5.2.

Step 0. Let $M=A$. Let $k=n$. Let $\rho=+\infty$.

Step 1. If $M$ is not a Z-matrix, stop; A cannot be an MMA-matrix.

Step D. For each of the $\left(\begin{array}{c}k \\ 2\end{array}\right) 2 \times 2$ principal submatrices $M[\{i, j\}]$ of $M$ (without loss, $i<j$ ), compute

$$
\lambda_{i j}=\frac{1}{2}\left[m_{i i}+m_{j j}+\left[\left(m_{i i}-m_{j j}\right)^{2}+4 m_{i j} m_{j i}\right]^{1 / 2}\right] .
$$

Step 3. Let $\lambda_{\max }=\max \left\{\lambda_{i j} \mid 1 \leq i<j \leq k\right\}$. If $\lambda_{\max }>\rho$, stop; $A$ cannot be an MMA-matrix.

Step 4. Select a pair $\{i, j\}$ such that $\lambda_{i j}=\lambda_{\max }$, and compute row and column eigenvectors $v^{t}$ and $u$, respectively, of $M[i, j]$.

Step 5. If either $M[(<k>\backslash\{i, j\}) \mid\{i, j\}] u \neq 0$ or $v^{t} M[\{i, j\} \mid(<k>\backslash\{i, j\})] \neq 0$, then $A$ cannot be an MMA-matrix. (If $<k>\backslash\{i, j\}$ is the empty set, then the equalities are trivially true.)

Step 6. Let $\mu_{k}=\lambda_{i j}$. If necessary, scale $u$ and $v$ so that $v^{t} u=1$. Let $\tilde{u}$ be the $k \times 1$ vector obtained from the $k \times 1$ vector of ones by replacing the $i^{\text {th }}$ and $j^{\text {th }}$ entries with $\left|u_{2}\right|$ and $\left|u_{1}\right|$, respectively. Let $\widetilde{v}$ be the $k \times 1$ vector obtained from the $k \times 1$ vector of ones by replacing the $i^{\text {th }}$ and $j^{\text {th }}$ entries with $\left|v_{2}\right|$ and $\left|v_{1}\right|$, respectively. Let $U_{k}$ be the $k \times k$ matrix $U_{k}=\widetilde{v} \widetilde{u}^{t}$. Let $\Pi$ be the $(k-1)$-partition of $k$ whose unique nonsingleton partition subset is $S_{1}=\{i, j\}$.

Step 7. Let $M=\left[A-\mu_{k} G(U)\right] / / U_{k}$. Let $\rho=\mu_{k}$. Assign $k=k-1$. If $k \geq 2$, go to Step 1.

Step 8. If $M=\left[m_{11}\right]$ has $0<m_{11}<\rho$, then $A$ is an $M M A$-matrix; let $\mu_{k}=$ $m_{11}$. Otherwise, $A$ is not an MMA-matrix. When $A$ is an MMA-matrix, the spectral decomposition is given by Theorem 3.2. 
Proof. Note that $A=M \times \times U+\rho(A) G(U)$ is an MMA-matrix if and only if $M$ is an MMA-matrix, $\rho(A) \geq \rho(M)$ and $U$ is a strictly positive inflator. Note that if either $A$ or $M$ is an MMA-matrix, then it is necessarily a Z-matrix, hence Step 1. Steps 2 through 5 follow from Theorem 4.1. Steps 6 and 7 are the result of Theorem 8.1 of [13]. Step 8 follows from the observation that $A$ is an MMA-matrix only if $M$ is an M-matrix, and from the nature of $1 \times 1 \mathrm{M}$-matrices.

Now we consider the operation count. The $(n-k+1)^{s t}$ iteration requires $O\left(k^{2}\right)$ multiplications and square roots during the production of the $\lambda_{i j}$. For one value of $\lambda_{i j}=\lambda_{\max }$, a pair of $2 \times 2$ linear systems must be solved to obtain the corresponding row and column eigenvectors, resulting in roughly sixteen multiplications. Testing the pair of eigenvectors to see if it extends to eigenvectors of the $k \times k$ matrix $M$, results in further $O(k)$ multiplications. If the pair of eigenvectors is extensible, then $U_{k}$ must be constructed, which requires $O\left(k^{2}\right)$ multiplications. Construction of $A-\mu_{k} G(U)$ requires four multiplications. The construction of $M$ as $\left[A-\mu_{k} G(U)\right] / / U_{k}$ requires $k^{2}$ divisions. Summing over $k$, it follows that there are $O\left(n^{3}\right)$ multiplications and $O\left(n^{3}\right)$ square roots required by the algorithm when $A$ is an MMA-matrix. Assuming that each square root requires at most $O(1)$ multiplications and divisions, the stated count is obtained. In addition to the multiplications and square roots, the algorithm requires a total of $O\left(n^{3}\right)$ comparisons. Finally, explicitly constructing each $E_{k}$ requires at most $O\left((n-k)^{2}\right)$ multiplications, so construction of the spectral decomposition requires $O\left(n^{3}\right)$ multiplications.

When $A$ is an MMA-matrix, the vectors $u$ and $v$ constructed during Step 6 of the algorithm are actually known to be unique subject to the following additional constraints: $u^{t} u=v^{t} v$, and the first entry of each of $u$ and $v$ are positive. Also, when $A$ is symmetric, it is always possible to choose $v=u$; see [13].

It was noted in [3] that every irreducible, $2 \times 2 \mathrm{M}$-matrix is an MMA-matrix. Consequently, it often suffices to continue a decomposition based on the previous theorem until the matrix $M$ obtained is $2 \times 2$, and then simply note whether or not $M$ is an M-matrix with negative off-diagonal entries.

It is worth noting that when exact arithmetic is employed, the algorithms in this section and the next produce the true spectral decompositions, not approximations, for (inverse) MMA-matrices of arbitrarily large size in a finite number of steps.

6. Testing for Inverse MMA-matrices. The following results naturally mirror those of the previous section.

THEOREM 6.1. The following algorithm determines whether the given $n \times n$, real matrix $A$ is an inverse MMA-matrix. The algorithm requires at most $O\left(n^{3}\right)$ multiplications and divisions, and at most $O\left(n^{3}\right)$ comparisons, and when $A$ is an inverse MMA-matrix, the algorithm produces the spectral decomposition of $A$.

AlgoRithm 6.2.

Step 0. Let $M=A$. Let $k=n$. Let $\rho=0$.

Step 1. If $M$ is not a strictly positive matrix, stop; A cannot be an inverse MMAmatrix.

Step 2. For each of the $\left(\begin{array}{c}k \\ 2\end{array}\right) 2 \times 2$ principal submatrices $M[\{i, j\}]$ of $M$ (without loss, 
$i<j)$, compute

$$
\lambda_{i j}=\frac{1}{2}\left[m_{i i}+m_{j j}-\left[\left(m_{i i}-m_{j j}\right)^{2}+4 m_{i j} m_{j i}\right]^{1 / 2}\right] .
$$

Step 3. Let $\lambda_{\min }=\min \left\{\lambda_{i j} \mid 1 \leq i<j \leq k\right\}$. If $\lambda_{\min }<\rho$, stop; $A$ cannot be an inverse MMA-matrix.

Step 4. Select a pair $\{i, j\}$ such that $\lambda_{i j}=\lambda_{\min }$, and compute row and column eigenvectors $v^{t}$ and $u$, respectively, of $M[i, j]$.

Step 5. If either $M[<k>\backslash\{i, j\} \mid\{i, j\}] u \neq 0$ or $v^{t} M[\{i, j\} \mid<k>\backslash\{i, j\}] \neq 0$, then $A$ cannot be an inverse MMA-matrix. (If $<k>\backslash\{i, j\}$ is the empty set, then the equalities are trivially true.)

Step 6. Let $\mu_{k}=\lambda_{i j}$. If necessary, scale $u$ and $v$ so that $v^{t} u=1$. Let $\tilde{u}$ be the $k \times 1$ vector obtained from the $k \times 1$ vector of ones by replacing the $i^{\text {th }}$ and $j^{\text {th }}$ entries with $\left|u_{2}\right|$ and $\left|u_{1}\right|$, respectively. Let $\widetilde{v}$ be the $k \times 1$ vector obtained from the $k \times 1$ vector of ones by replacing the $i^{\text {th }}$ and $j^{\text {th }}$ entries with $\left|v_{2}\right|$ and $\left|v_{1}\right|$, respectively. Let $U_{k}$ be the $k \times k$ matrix $U_{k}=\widetilde{v} \widetilde{u}^{t}$. Let $\Pi$ be the $(k-1)$-partition of $k$ whose unique nonsingleton partition subset is $S_{1}=\{i, j\}$.

Step 7. Let $M=\left[A-\mu_{k} G(U)\right] / / U_{k}$. Let $\rho=\mu_{k}$. Assign $k=k-1$. If $k \geq 2$, go to Step 1.

Step 8. If $M=\left[m_{11}\right]$ has $m_{11}>\rho$, then $A$ is an inverse $M M A$-matrix; let $\mu_{k}=m_{11}$. Otherwise, $A$ is not an inverse MMA-matrix. When $A$ is an inverse MMA-matrix, the spectral decomposition of $A$ is given by

$$
A=\sum_{k=1}^{n} \mu_{k} E_{k},
$$

where the $E_{k}$ are given by Theorem 3.2.

Proof. This result follows from applying Theorem 2.1 and part (iii) of Lemma 3.3 to the theorem in the previous section, and noting that $A$ is an MMA-matrix with maximal eigenvalue $\lambda$ exactly when $A^{-1}$ is an inverse MMA-matrix with minimal eigenvalue $\lambda^{-1}$. Note that Step 1 is simply the requirement that $M$ be the inverse of an irreducible M-matrix.

7. Testing for Soules Bases. In this section, $A$ is required to be symmetric.

Elsner, Nabben and Neumann prove in Observation 2.1 of [1] that the $n \times n$, real matrix $R$ is a Soules matrix if and only if the following three conditions on its columns hold: (i) $r_{1}$ is strictly positive; (ii) for $1 \leq h<n, \sum_{k=1}^{h} r_{k} r_{k}^{t}$ is entrywise nonnegative; and (iii) $\sum_{k=1}^{n} r_{k} r_{k}^{t}=I_{n}$. Fielder proves in Theorem 5 of [2] that if the real matrix $A$ with spectrum satisfying $0<\alpha_{1}<\alpha_{2} \leq \cdots \leq \alpha_{n}$ has spectral decomposition

$$
A=\sum_{k=1}^{n} \alpha_{k} E_{k},
$$


where $E_{1}$ is strictly positive, $\sum_{k=1}^{h} E_{k}$ is entrywise nonnegative for $1 \leq h<n$, and $\sum_{k=1}^{n} E_{k}=I_{n}$, then $A$ must be an MMA-matrix. Theorem 3.2 in turn implies that the sequence $\left\{E_{k}\right\}_{k=1}^{n}$ of spectral projections must be inflation-generated by a sequence of strictly positive, rank one inflators $\left\{U_{k}\right\}_{k=1}^{n}$, and when each $E_{k}$ is symmetric, then so is each $U_{k}$. Finally, set $E_{k}=r_{k} r_{k}^{t}$ for each $k$. Thus:

THEOREM 7.1. Let $R$ be an $n \times n$ real matrix with columns $r_{1}, r_{2}, \ldots, r_{n}$. Then $R$ is a Soules matrix if and only if $\left\{r_{k} r_{k}^{t}\right\}_{k=1}^{n}$ is inflation-generated by a sequence of strictly positive, rank one inflators $\left\{U_{k}\right\}_{k=1}^{n}$.

Rather than forming each of the matrices $r_{k} r_{k}^{t}$, selecting a set of eigenvalues $\alpha_{k}$, building the MMA-matrix $A$, and then applying Algorithm 5.2 in order to recover the inflation sequence, it is possible to test the columns of $R$ directly using inflation products of vectors. This provides the following alternative test to that given by Theorem 2.2 of [1].

THEOREM 7.2. Let $R$ be an $n \times n$ real, orthogonal matrix with columns $r_{1}, r_{2}$, $\ldots, r_{n}$. Suppose that $r_{1}$ is strictly positive and that $n \geq 2$. The following algorithm determines whether $R$ is a Soules matrix. The algorithm requires $O\left(n^{2}\right)$ divisions, no multiplications, and no square roots.

Algorithm 7.3 .

Step 0. Set $P=R$. Label the columns of $P$ as $p_{1}, p_{2}, \ldots, p_{n}$. Let $k=n$.

Step 1. If $p_{k}$ does not have exactly one positive and exactly one negative entry, then $R$ is not a Soules matrix.

Step 2. Let $i$ and $j$, with $i<j$, be the indices of the nonzero entries of $p_{k}$, and label those entries $\alpha_{k}$ and $\beta_{k}$, respectively.

Step 3. Let $\widetilde{u}_{k}$ be the $k \times 1$ vector obtained from the vector of all ones by replacing the $i^{\text {th }}$ entry by $\left|\beta_{k}\right|$ and the $j^{\text {th }}$ entry by $\left|\alpha_{k}\right|$.

Step 4. Let $\Pi_{k}$ be the $(k-1)$-partition of $k$ whose unique nonsingleton partition subset is $\{i, j\}$.

Step 5. Let $P$ be the $(k-1) \times(k-1)$ matrix whose columns are $p_{1} / / \widetilde{u}_{k}, p_{2} / / \widetilde{u}_{k}, \ldots$, $p_{k-1} / / \widetilde{u}_{k}$.

Step 6. Assign $k=k-1$. If $k \geq 2$, go to Step 1 .

Step 7. If $k=1$, then $R$ is a Soules matrix.

Proof. Suppose $P$ is a $k \times k$ Soules matrix. Then $p_{k} p_{k}^{t}=G\left(U_{k}\right)$ for a strictly positive, rank one inflator $U_{k}$ by Theorem 7.1, and hence $p_{k}$ must have exactly one positive and exactly one negative entry. Since $p_{k}$ is orthogonal to every other column of $P$, it follows that the $i, j$ subvector of every other column must be a scalar multiple of $\left(\left|\beta_{k}\right|,\left|\alpha_{k}\right|\right)^{t}$, and hence, $p_{h} / / \widetilde{u}_{k}$ is a well-defined, normal vector for $1 \leq h<k$. Furthermore, orthogonality is preserved among the $p_{h} / / \widetilde{u}_{k}$ since $\alpha_{k}^{2}+\beta_{k}^{2}=1$. Finally, notice that if $P$ is a Soules matrix, then each $p_{h} p_{h}^{t}=E_{h}$, which is an inflation-generated spectral projection, and hence $E_{h} / / U_{h}$ is also an inflation-generated spectral projection, where $U_{k}=\widetilde{u}_{k} \tilde{u}_{k}^{t}$. It is easy to check that $E_{h} / / U_{h}=\left(p_{h} / / \widetilde{u}_{k}\right)\left(p_{h} / / \widetilde{u}_{k}\right)^{t}$. If $R$ is a Soules matrix, it follows that each $P$ must be a Soules matrix.

It follows from the decomposition of $R$ in the preceding algorithm that if $R$ is a Soules matrix, then the columns of $R$ are $(1) \times \times \widetilde{u}_{2} \times \times \widetilde{u}_{3} \times \times \cdots \times \times \widetilde{u}_{n}, p_{2} \times \times \widetilde{u}_{3} \times$ 
$\times \cdots \times \times \widetilde{u}_{n}, \ldots, p_{n-1} \times \times \widetilde{u}_{n}, r_{n}$. Note that the decomposition in Algorithms 5.2 and 6.2 actually provide the vectors needed to generate the corresponding Soules matrix. Also note that the algorithm uses no multiplications, and that divisions occur only in Step 5. Each computation of a vector $p_{h} / / \widetilde{u}_{k}$ requires exactly two divisions since all but two entries of the vector $\widetilde{u}_{k}$ are ones. Thus the $(n-k+1)^{s t}$ iteration requires $2(k-1)$ divisions.

8. Testing for Strictly Ultrametric Matrices. In this section, $A$ is required to be symmetric.

Observe that testing whether the $n \times n$ matrix $A$ is strictly ultrametric can be done using only comparisions, and requires at most $O\left(n^{3}\right)$ comparisons. In light of Theorem 2.3, it would be possible to test whether $A$ is a strictly ultrametric matrix by applying Algorithm 6.2 to $F A F$. Note, however that the spectral decomposition of $F A F$ will not generally be closely related to that of $A$. One case in which the spectral decomposition of $F A F$ would be useful is when all row sums of $A$ are equal, with common sum $r$. In this case, $p=r e, F=\frac{1}{\sqrt{r}} I_{n}$, and the spectral decomposition of $A$ is given by

$$
A=\sum_{k=1}^{n} r \mu_{k} E_{k}
$$

where the $\mu_{k}$ and the $E_{k}$ are those produced as the final output of Algorithm 6.2 applied to $F A F$.

9. An Example of Spectral Decomposition. Let $A$ be the following $3 \times 3$, real, symmetric matrix:

$$
A=\left[\begin{array}{ccc}
\frac{601}{338} & \frac{90}{169} & \frac{12}{13} \\
\frac{90}{169} & \frac{122}{169} & \frac{5}{13} \\
\frac{12}{13} & \frac{5}{13} & 2
\end{array}\right]
$$

Is $A$ an inverse MMA-matrix? Apply Algorithm 6.2. Let $M=A$. Let $k=3$. Let $\rho=0$. Computing, $\lambda_{12}=0.5000, \lambda_{13}=0.6151, \lambda_{23}=0.9523 . \lambda_{\min }=0.5000>\rho$. Then $u=v=\left(\frac{5}{13},-\frac{12}{13}\right)^{t}$. Testing, $M[\{3\} \mid\{1,2\}] u=\left[\begin{array}{cc}\frac{12}{13} & \frac{5}{13}\end{array}\right] u=(0,0)^{t}$. Then $\widetilde{u}=\left(\frac{12}{13}, \frac{5}{13}, 1\right)^{t}$ and $\Pi_{3}=\{\{1,2\},\{3\}\}$. Hence,

$$
U_{3}=\left[\begin{array}{ccc}
\frac{144}{169} & \frac{60}{169} & \frac{12}{13} \\
\frac{60}{169} & \frac{25}{169} & \frac{5}{13} \\
\frac{12}{13} & \frac{5}{13} & 1
\end{array}\right] \text {, and } G\left(U_{3}\right)=\left[\begin{array}{ccc}
\frac{25}{169} & -\frac{60}{169} & 0 \\
-\frac{60}{169} & \frac{144}{169} & 0 \\
0 & 0 & 0
\end{array}\right]
$$

With $\mu_{3}=0.5000$,

$$
M-\mu_{3} G\left(U_{3}\right)=\left[\begin{array}{ccc}
\frac{288}{169} & \frac{120}{169} & \frac{12}{13} \\
\frac{120}{169} & \frac{50}{169} & \frac{5}{13} \\
\frac{12}{13} & \frac{5}{13} & 2
\end{array}\right]=\left[\begin{array}{ll}
2 & 1 \\
1 & 2
\end{array}\right] \times \times U_{3} .
$$


Set $M=\left[\begin{array}{ll}2 & 1 \\ 1 & 2\end{array}\right], k=2$, and $\rho=0.5000$. Then $\lambda_{\min }=\lambda_{12}=1 \geq \rho$, and $u=v=\frac{1}{\sqrt{2}}(1,-1)^{t}$. Then $\tilde{u}=\frac{1}{\sqrt{2}}(1,1)^{t}$; and $\Pi_{2}=\{\{1,2\}\}$. Hence,

$$
U_{2}=\frac{1}{2}\left[\begin{array}{ll}
1 & 1 \\
1 & 1
\end{array}\right] \text { and } G\left(U_{2}\right)=\frac{1}{2}\left[\begin{array}{cc}
1 & -1 \\
-1 & 1
\end{array}\right] .
$$

With $\mu_{2}=1$,

$$
M-\mu_{2} G\left(U_{2}\right)=\frac{1}{2}\left[\begin{array}{ll}
3 & 3 \\
3 & 3
\end{array}\right]=[3] \times \times U_{2} .
$$

Set $k=1$ and $\rho=1$. Since $m_{11}=3>\rho$, it follows that $A$ is an inverse MMA-matrix. Set $\mu_{1}=3$.

Finally, the spectral decomposition of $A$ is $A=\sum_{k=1}^{3} \mu_{k} E_{k}$, where $E_{3}=G\left(U_{3}\right)$,

$$
E_{2}=G\left(U_{2}\right) \times \times U_{3}=\frac{1}{2}\left[\begin{array}{ccc}
\frac{144}{169} & \frac{60}{169} & -\frac{12}{13} \\
\frac{60}{169} & \frac{25}{169} & -\frac{5}{13} \\
-\frac{12}{13} & -\frac{5}{13} & 1
\end{array}\right],
$$

and

$$
E_{1}=G\left(U_{1}\right) \times \times U_{2} \times \times U_{3}=\frac{1}{2}\left[\begin{array}{ccc}
\frac{144}{169} & \frac{60}{169} & \frac{12}{13} \\
\frac{60}{169} & \frac{25}{169} & \frac{5}{13} \\
\frac{12}{13} & \frac{5}{13} & 1
\end{array}\right]
$$

since $G\left(U_{1}\right)$ is the identity matrix by definition. Notice that it is possible to recover the Soules basis $\left\{r_{1}, r_{2}, r_{3}\right\}$, since $E_{k}=r_{k} r_{k}^{t}$. Thus the Soules basis here is $\left\{\frac{1}{\sqrt{2}}\left(\frac{12}{13}, \frac{5}{13}, 1\right)^{t}, \frac{1}{\sqrt{2}}\left(\frac{12}{13}, \frac{5}{13},-1\right)^{t},\left(\frac{5}{13},-\frac{12}{13}, 0\right)^{t}\right\}$. If the vectors $u$ produced during the algorithm are labelled as $u_{3}=\left(\frac{5}{13},-\frac{12}{13}\right)^{t}$ and $u_{2}=\frac{1}{\sqrt{2}}(1,-1)^{t}$; their embeddings into the all zeros vectors are labelled $p_{3}=\left(\frac{5}{13},-\frac{12}{13}, 0\right)^{t}$ and $p_{2}=\frac{1}{\sqrt{2}}(1,-1)^{t}$ and the vectors $\widetilde{u}$ produced by the algorithm are labelled as $\widetilde{u}_{3}=\left(\frac{12}{13}, \frac{5}{13}, 1\right)^{t}$ and $\tilde{u}_{2}=\frac{1}{\sqrt{2}}(1,1)^{t}$, then the Soules basis is obtained as the following set:

$\left\{(1) \times \times \widetilde{u}_{2} \times \times \widetilde{u}_{3}, p_{2} \times \times \widetilde{u}_{3}, p_{3}\right\}$.

10. A Cautionary Example. Let $B$ be the matrix

$$
B=\frac{1}{3}\left[\begin{array}{lll}
5 & 6 & 4 \\
6 & 9 & 6 \\
4 & 6 & 5
\end{array}\right]
$$

Then

$$
B=\left[\begin{array}{cc}
3 & 2 \sqrt{2} \\
2 \sqrt{2} & 3
\end{array}\right] \times \times U_{3}+\lambda_{13} G\left(U_{3}\right)
$$


where

$$
U_{3}=\frac{1}{2}\left[\begin{array}{ccc}
1 & \sqrt{2} & 1 \\
\sqrt{2} & 2 & \sqrt{2} \\
1 & \sqrt{2} & 1
\end{array}\right]
$$

is a strictly positive, rank one inflator with ordered partition $\Pi=\{\{1,3\},\{2\}\}$,

$$
G\left(U_{3}\right)=\frac{1}{2}\left[\begin{array}{ccc}
1 & 0 & -1 \\
0 & 0 & 0 \\
-1 & 0 & 1
\end{array}\right] ;
$$

and $\lambda_{13}=\frac{1}{3}$ comes from $B[\{1,3\}]$. Furthermore,

$$
\left[\begin{array}{cc}
3 & 2 \sqrt{2} \\
2 \sqrt{2} & 3
\end{array}\right]
$$

is an inverse MMA-matrix. Computing,

$$
B^{-1}=\left[\begin{array}{ccc}
3 & -2 & 0 \\
-2 & 3 & -2 \\
0 & -2 & 3
\end{array}\right],
$$

which is an M-matrix; however,

$$
\left(B^{-1}\right)^{2}=\left[\begin{array}{ccc}
13 & -12 & 4 \\
-12 & 17 & -12 \\
4 & -12 & 13
\end{array}\right],
$$

which is not an M-matrix. Thus, $B$ is not an inverse MMA-matrix even though it appears to have the inflation-based decomposition. The reason is that $\lambda_{13} \neq \lambda_{\min }$ in Step 3 of Algorithm 6.2. Indeed, $\lambda_{13}=1 / 3$ is not the minimum eigenvalue of $B$ since $\operatorname{spec}(B)=\{0.1716,0.3333,5.8284\}$. In fact, $\lambda_{12}=\lambda_{23}=\lambda_{\min }=0.2251<\lambda_{13}$, and neither of the vectors $u=v$ constructed for $\lambda_{12}$ nor for $\lambda_{23}$ pass the test in Step 5 of the algorithm; thus the algorithm detects that $B$ is not an inverse MMA-matrix.

11. Extending Soules Bases. It is possible to use inflation and the graph theoretic structures in [10] and [14] to give both an alternative construction to the one given by Elsner, Nabben and Neumann in [1] for all possible Soules matrices that arise from a given strictly positive vector $r$ of unit length and to give an alternative combinatorial analysis of the zero nonzero patterns involved. We give here the fundamental extension result.

THEOREM 11.1. Let $R$ be an $n \times n$ Soules matrix with columns $r_{1}, r_{2}, \ldots, r_{n}$. Choose an index $i$ with $1 \leq i \leq n$, choose indices $j_{1}$ and $j_{2}$ with $1 \leq j_{1}<j_{2} \leq n+1$, and choose an $\alpha$ with $0<\alpha<1$. Let $u$ be obtained from the $(n+1) \times 1$ vector of ones by replacing the $j_{1}$ entry with $\sqrt{\alpha}$ and the $j_{2}$ entry with $\sqrt{1-\alpha}$. Let $\Pi$ be the n-partition of $(n+1)$ whose only nonsingleton block is $B_{i}=\left\{j_{1}, j_{2}\right\}$, and whose singleton blocks are the entries of $\{1,2, \ldots, n+1\} \backslash\left\{j_{1}, j_{2}\right\}$ in increasing order. For 
$1 \leq h \leq n$, define $t_{h}=r_{h} \times \times u$, and let $t_{n+1}$ be the $(n+1) \times 1$ vector obtained from the zero vector by replacing the $j_{1}$ entry with $\sqrt{1-\alpha}$ and the $j_{2}$ entry with $-\sqrt{\alpha}$. Then the matrix $T$ whose columns are the vectors $t_{h}$ is an $(n+1) \times(n+1)$ Soules matrix.

Proof. Clearly, one iteration of Algorithm 7.3 applied to $T$ will yield $R$, which is known to be a Soules matrix.

Note that the choice of the signs in the entries of $t_{n+1}$ can be reversed. Furthermore, it can be shown that up to the choice of signs and up to the permutation of the singleton blocks, these are the only extensions of $R$ in the sense of recovering $R$ from the decomposition given in Algorithm 7.3. Intuitively, what the algorithm does is replace the $i^{t h}$ entry of the $h^{t h}$ column of $R$ with a pair of entries: $\left(r_{h}\right)_{i} \sqrt{\alpha}$ and $\left(r_{h}\right)_{i} \sqrt{1-\alpha}$, placing them in positions $j_{1}$ and $j_{2}$, and distributing the remaining entries of $h^{\text {th }}$ column of $R$ in the remaining positions. It should be obvious that this preserves orthonormality of the columns. The final column is just the embedding of $(\sqrt{1-\alpha},-\sqrt{\alpha})^{t}$ into the vector of zeros. Clearly it is normal and also orthogonal to the first $n$ columns.

EXAMPLE 11.2. Let $R$ be the matrix whose columns are $\frac{1}{\sqrt{2}}\left(\frac{12}{13}, \frac{5}{13}, 1\right)^{t}$,

$\frac{1}{\sqrt{2}}\left(\frac{12}{13}, \frac{5}{13},-1\right)^{t}$, and $\left(\frac{5}{13},-\frac{12}{13}, 0\right)^{t}$ from Section 9. Let $i=2$, let $j_{1}=1$, let $j_{2}=3$, and let $\alpha=\frac{9}{25}$. Let $\Pi$ be the 3 -partition of 4 given by $B_{1}=\{2\}, B_{2}=\{1,3\}$, and $B_{3}=$ $\{4\}$. Then $u=\left(\frac{3}{5}, 1, \frac{4}{5}, 1\right)^{t}$. Furthermore, $T$ has columns $\frac{1}{\sqrt{2}}\left(\frac{5}{13} \cdot \frac{3}{5}, \frac{12}{13}, \frac{5}{13} \cdot \frac{4}{5}, 1\right)^{t}$, $\frac{1}{\sqrt{2}}\left(\frac{5}{13} \cdot \frac{3}{5}, \frac{12}{13}, \frac{5}{13} \cdot \frac{4}{5},-1\right)^{t},\left(-\frac{12}{13} \cdot \frac{3}{5}, \frac{5}{13},-\frac{12}{13} \cdot \frac{4}{5}, 0\right)^{t}$ and $\left(\frac{4}{5}, 0,-\frac{3}{5}, 0\right)^{t}$.

Acknowledgement. The author wishes to thank Professor Hans Schneider for introducing him to the subjects of inflation and MMA-matrices.

\section{REFERENCES}

[1] L. Elsner, R. Nabben, and M. Neumann. Orthogonal bases that lead to symmetric, nonnegative matrices. Linear Algebra Appl., 271:323-343, 1998.

[2] M. Fiedler. Characterization of MMA-matrices. Linear Algebra Appl., 106:233-244, 1988.

[3] S. Friedland, D. Hershkowitz, and H. Schneider. Matrices whose powers are M-matrices or Z-matrices. Trans. Amer. Math. Soc., 300:343-366, 1987.

[4] D. Hershkowitz and S. Schneider. Matrices with a sequence of accretive powers. Israel J. Math., $55: 344-372,1986$.

[5] S. Martínez, G. Michon, and J. San Martín. Inverses of ultrametric matrices of Stieltjes Type. SIAM J. Matrix Anal. Appl., 15:98-106, 1994.

[6] J. J. McDonald, M. Neumann, H. Schneider, and M. J. Tsatsomeros. Inverse M-matrix inequalities and generalized ultrametric matrices. Linear Algebra Appl., 220:321-341, 1995.

[7] R. Nabben and R. S. Varga. A linear algebra proof of the inverse of a strictly ultrametric matrix is a strictly diagonally dominant Stieltjes matrix. SIAM J. Matrix Anal. Appl., 15:107-113, 1994.

[8] R. Nabben and R. S. Varga. Generalized ultrametric matrices - a class of inverse M-matrices. Linear Algebra Appl., 220:365-390, 1995.

[9] G. W. Soules. Constructing symmetric, nonnegative matrices. Linear and Multilinear Algebra, 13:241-251, 1983.

[10] H. Schneider and J. Stuart. Allowable spectral perturbations for ZME-matrices. Linear Algebra Appl, 111:63-118, 1988. 
[11] J. Stuart. Eigenvectors for inflation matrices and inflation-generated matrices. Linear and Multilinear Algebra, 22:249-265, 1987.

[12] J. Stuart. The decomposition of idempotents associated with inflators. Linear Algebra Appl., 97:171-184, 1987.

[13] J. Stuart. An eigenvector test for inflation matrices and ZME-matrices. SIAM J. Matrix Anal. A ppl., 10:520-532, 1989.

[14] J. Stuart. The partial order graph for a ZME-matrix. Linear Algebra Appl, 141:123-152, 1990. 